\title{
Efecto de la contaminación en la interpretación de superficies de fractura metálicas
}

\section{Effect of the environment on the interpretation of the metallic fracture surfaces}

\author{
Fernando Iván Medina-Alcína \\ Ing. Metalúrgico \\ Instituto Universitario Tecnología \\ Puerto Cabello, Venezuela \\ fernandoivanmedina@hotmail.com
}

\begin{abstract}
Resumen- La contaminación de una superficie de fractura a causa del medio ambiente es perjudicial, ya que mucha información vital se pierde debido a la presencia de suciedad, productos de oxidación, humedad, etc., que se alojan en esta, pudiendo dar indicaciones erróneas de un evento de falla. Por lo anterior, la preservación de las piezas es un factor crítico en el análisis de falla. En esta investigación (4) muestras de acero ASTM A-36 fracturadas por impacto a temperatura ambiente, fueron colocadas a la intemperie en la ciudad de Puerto Cabello, Venezuela, por un período de tiempo de 6 meses. Luego se realizó una evaluación de la superficie de fractura corroída, con una lupa estereoscópica, para observar el crecimiento de los productos de corrosión. Los resultados obtenidos indican que toda la información relacionada con el proceso de fractura en general, se puede perder en pocas semanas a causa del impacto ambiental.
\end{abstract}

Palabras clave- Fractografía, limpieza ultrasónica, manejo de fracturas.

\begin{abstract}
Contamination of metallic fractures is detrimental, since the information related with the fracture process can be lost. As rust, humidity, foreign debris, etc., on metallic fracture surfaces can give false information about a failure event, the preservation of fractured samples is critical for failure analysis. In this investigation four (4) impact fracture surfaces of ASTM A-36 steel were placed outdoor in Puerto Cabello, Venezuela, for a period of 6 months. Then, an evaluation of the fractured surfaces was performed, using a stereoscopic magnifying glass, to observe the growth of corrosion products. The results indicate that the information related to the general fracture process can be lost because of the environmental impact.
\end{abstract}

Keywords - Fractography, ultrasonic cleaning, fracture management.

\section{INTRODUCCIÓN}

Una fractura contiene información vital acerca de los mecanismos involucrados durante un evento de falla, preservar esa información es crucial en las primeras horas posteriores a la falla, ya que el medio ambiente degradará la superficie de fractura inmediatamente ocurrida esta. Sin embargo, ciertos depósitos y suciedad en la superficie de fractura pueden contener información que es vital para comprender la causa de la falla.

Primeramente se debe considerar si los productos de corrosión son importantes para comprender la falla, se debe también documentar y dejar establecida toda la información posible de la pieza, incluyendo la fotografía [1] acompañada además de una evaluación de la superficie con lupa estereoscópica, si los productos de corrosión limitan la observación de la superficie de fractura estos deben ser removidos antes de iniciar la investigación de las causas que originaron la falla como tal. Los procedimientos de limpieza deben ser igualmente supervisados y la superficie cuidadosamente preservada [2]; en caso contrario, la contaminación compromete seriamente el desarrollo de la investigación [3], [4], como se puede apreciar en la Fig. 1, en la cual un eje agitador construido de una aleación AISI 904L y cuya función dentro del proceso es agitar el fluido (una solución de ácido fosfórico al 28\% y 30\% de sólidos) que contiene el reactor para favorecer las 
reacciones y mantener en suspensión los sólidos, presentó una falla que al principio se asoció a corrosión por picaduras.

Fig. 1. SUPERFICIE DE FRACTURA DEL EJE ROTOR DEL REACTOR

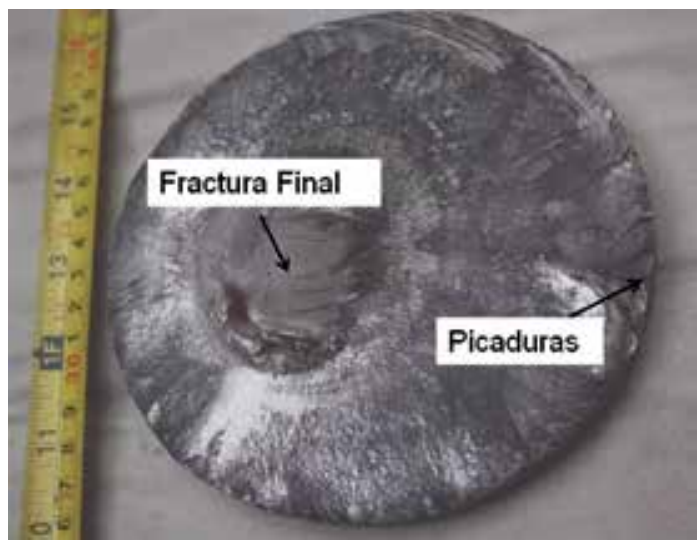

Fuente: autor.

La pieza fracturada fue almacenada en el taller de la empresa, mientras que la otra sección del eje fracturado fue reparada y puesta en servicio. Cuatro meses después, la empresa decide investigar las razones de falla del eje agitador y realiza el corte del mismo obteniéndose un disco de aproximadamente $16 \mathrm{~cm}$ de diámetro, el cual es enviado al laboratorio metalográfico del Instituto Universitario de Tecnología Puerto Cabello (IUTPC), allí se procedió a realizar un registro documental de la pieza y se realizó su corte para obtener las muestras que fueron enviadas al laboratorio de microscopía electrónica del Instituto Universitario de Tecnología Región Capital Dr. Federico Rivero Palacios (IUTRC) en la ciudad de Caracas, para el análisis superficial del área donde se presentaron las picaduras y la zona de fractura final.

Los primeros resultados obtenidos de la evaluación con el microscopio electrónico (Fig. 2) muestran una fractografía (microscopía electrónica de barrido, MEB), donde se pueden observar algunos productos de corrosión sobre las estriaciones de fatiga. Una observación de la misma zona con lupa estereoscópica reveló la presencia de suciedad además de los productos de corrosión (Fig. 3).

La contaminación de la muestra influyó en los resultados obtenidos, ya que la información relacionada con la superficie de fractura quedó debajo de capas de óxido y suciedad, esta última más relacionada con las condiciones de almacenaje de la muestra que ambientales, una posterior limpieza ultrasónica y re-evaluación de esta zona con el microscopio electrónico revelaron con mayor detalle las estriaciones de fatiga (Fig. 4). Se han reportado recomendaciones para la preparación y preservación de muestras para microscopía electrónica y los efectos de la contaminación en los resultados, ya que es vital para esta técnica preservar de 1 a $20 \mathrm{~nm}$ que es propensa a la contaminación [5].

Aunque parezca algo secundario para un analista de fallas, siempre se debe manipular una fractura con mucho cuidado. El uso de guantes es recomendado, ya que se ha reportado que la contaminación de una superficie de fractura no depende necesariamente del medio ambiente. El mismo cuerpo humano es un ente corrosivo que puede transferir contaminantes, tales como humedad y sales que atacan químicamente el material con solo tocar la pieza [6].

Fig. 2. SUPERFICIE DE FRACTURA DEL EJE ROTOR DEL REACTOR BAJO MICROSCOPIO ELECTRÓNICO SIN LIMPIEZA

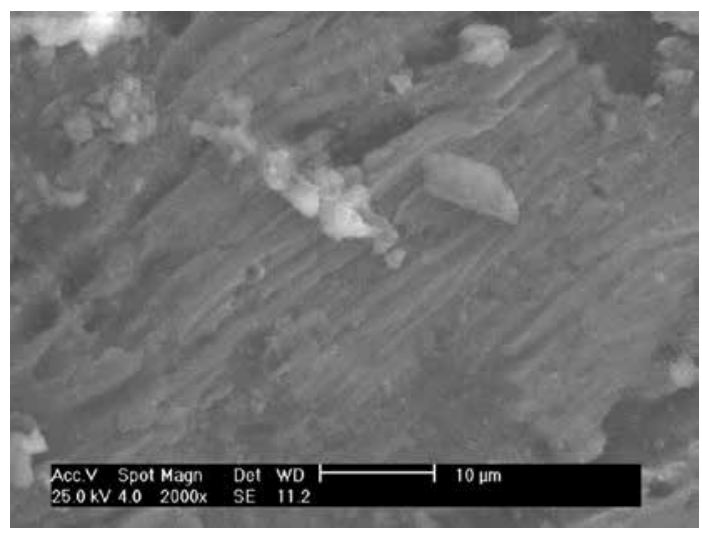

Fuente: autor.

Fig. 3. SUPERFICIE DE FRACTURA DEL EJE ROTOR DEL REACTOR BAJO LUPA ESTEREOSCÓPICA

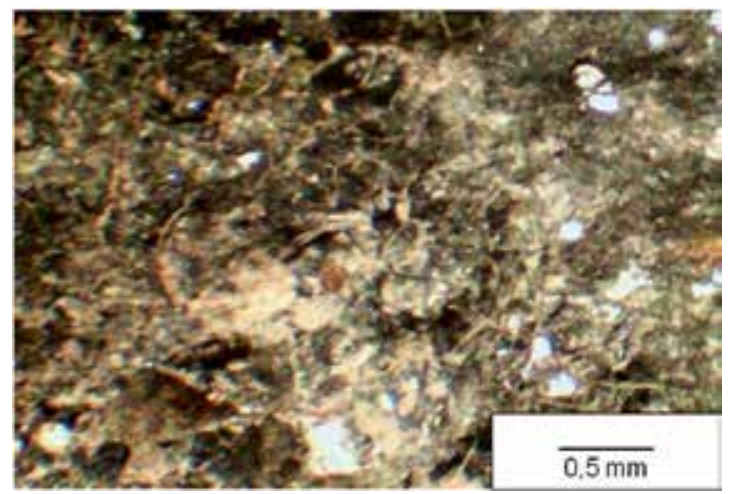

Fuente: autor. 
Fig. 4. SUPERFICIE DE FRACTURA DEL EJE AGITADOR DEL REACTOR BAJO MICROSCOPIO ELECTRÓNICO POSTERIOR A SU LIMPIEZA

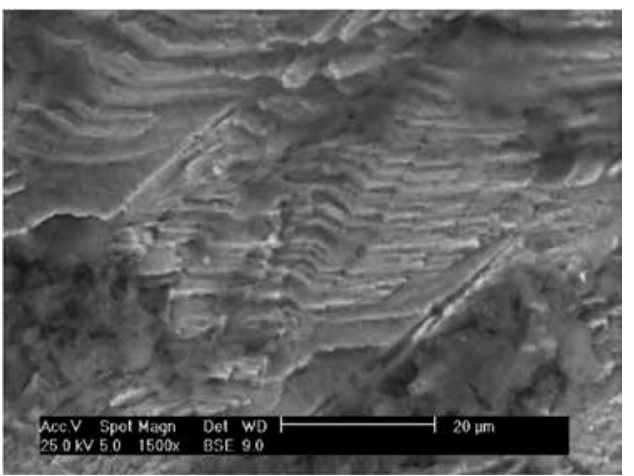

Fuente: autor.

\section{DESARROLLO}

\subsection{Procedimiento experimental}

Se utilizaron 4 probetas de impacto Charpy de acero ASTM A-36, preparadas de acuerdo con la norma ASTM E 23-82 [7], posterior a su preparación se procedió a su fractura en un péndulo con una energía de impacto de 300 Joules. Una vez fracturadas las probetas a temperatura ambiente, se realizó una selección de las superficies de fractura para su posterior análisis. Dos (2) de las muestras seleccionadas se colocaron en la zona marina de Puerto Cabello (Venezuela), por un período de seis (6) meses (desde el mes de diciembre período de sequias de 2013 hasta el mes de junio de 2014 período de lluvias), con la finalidad de inducir daño por corrosión en las respectivas superficies de fractura. Las dos (2) muestras restantes permanecieron en condiciones de laboratorio. Luego de seis (6) meses las superficies de fractura se analizaron con una lupa estereoscópica Amscope, acoplada a una cámara digital y un software Carl Zeiss.

\section{RESULTADOS Y DISCUSIÓN}

\subsection{Evaluación con lupa estereoscópica de muestras patrón}

En la Fig. 5 se pueden observar las muestras de acero ASTM A-36 que fueron empleadas en la investigación, estas se fracturaron por impacto a temperatura ambiente y posteriormente, se colocaron dos muestras en el ambiente de laboratorio (muestras 1 y 3 ) y las restantes (muestras 2 y 4 ) en los alrededores de la zona marina de Puerto Cabello, Venezuela; específicamente en las instalaciones del IUTPC. Una vez cumplido los 6 meses se procedió al retiro de las mismas y a evaluar su condición superficial. La evaluación consistió en una observación visual seguida de una con lupa estereoscópica; de esta inspección se encontró que las muestras 2 y 4 presentaban sus superficies de fracturas muy oxidadas, como era de esperarse por estar expuestas directamente al ambiente marino en comparación con las que estaban almacenadas en el laboratorio.

Fig. 5. MUESTRAS DE ACERO ASTM A-36 FRACTURADAS POR IMPACTO

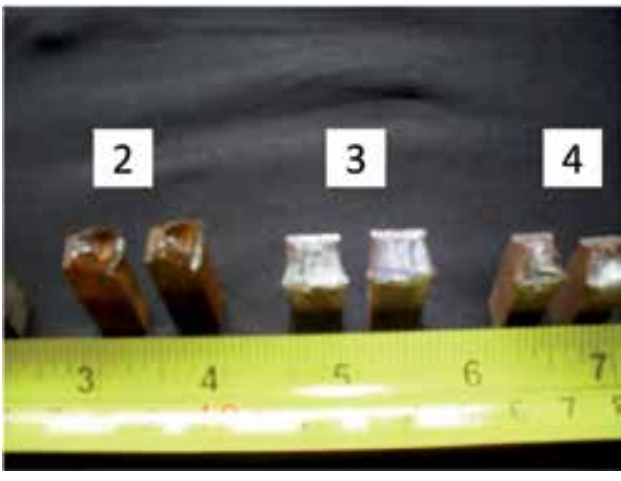

Fuente: autor.

Como lo muestran las Fig. 6 y Fig. 7, las superficies de fractura presentaron oxidación localizada, mientras que el resto aún conservaban su aspecto brillante. La presencia de estas zonas se debe al hecho de la rugosidad superficial de la fractura, ya que al momento de ocurrir esta, los planos de clivaje pueden dejar ciertas áreas con una alta rugosidad, por lo que son zonas muy activas para el intercambio de electrones entre el metal y el medio ambiente al cual está expuesto [8].

Fig. 6. SUPERFICIE DE FRACTURA DE ACERO PATRÓN ASTM A-36 CON OXIDACIÓN LOCALIZADA (FLECHA)

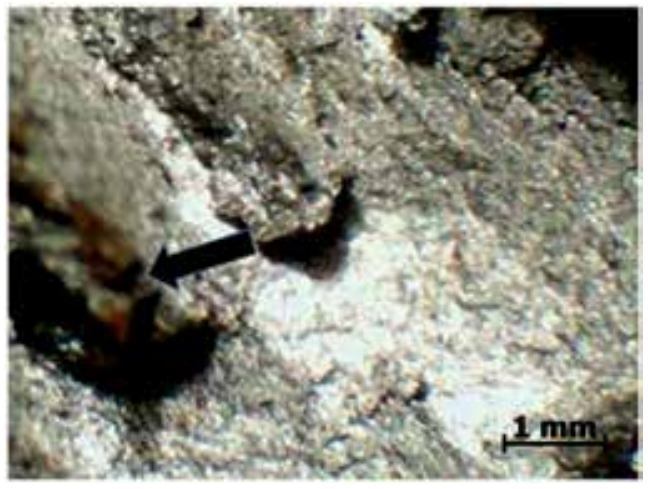

Fuente: autor. 
Fig. 7. AGRUPACIONES DE ÓXIDOS (FLECHA) SOBRE SUPERFICIE DE FRACTURA PATRÓN

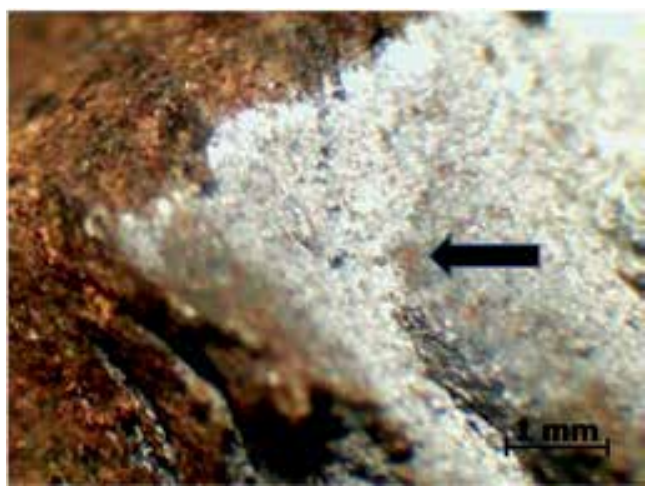

Fuente: autor.

\subsection{Evaluación con lupa estereoscópica de muestras colocadas en el ambiente marino de la ciudad de Puerto Cabello, Venezuela}

Como se puede observar en la Fig. 8, la oxidación fue acelerada por los factores ambientales de la zona marina de Puerto Cabello, los productos de corrosión ocultaron completamente la superficie de fractura.

Fig. 8. SUPERFICIE DE FRACTURA DE MUESTRA COLOCADA EN LAS INSTALACIONES DEL IUTPC PUERTO CABELLO
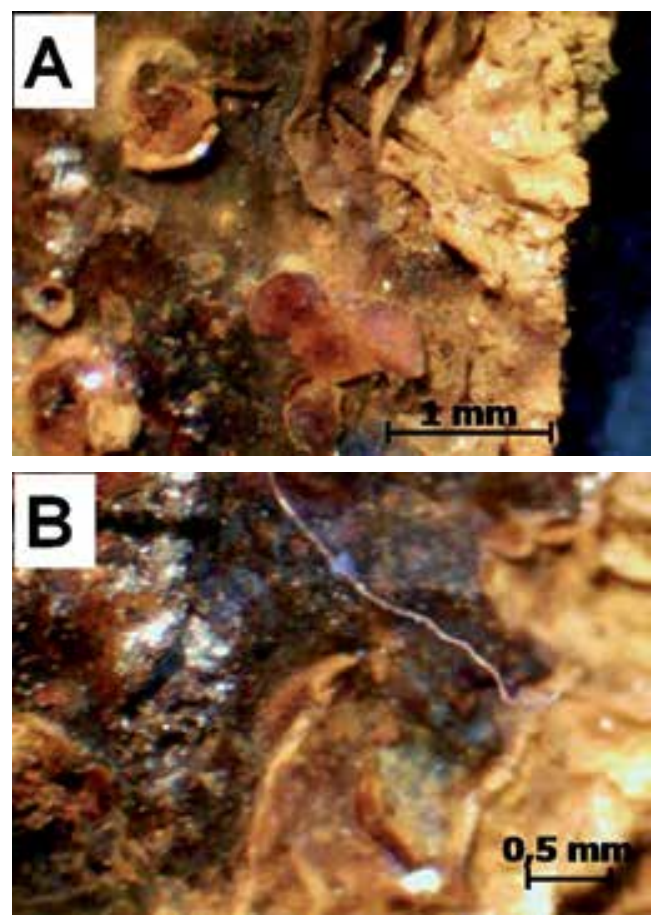

Fuente: autor

Este fenómeno se debe a que el medio ambiente aporta muchos elementos corrosivos, tales como: humedad, presencia de sales, iones cloruro, gases como $\mathrm{SO}_{2}$, y hasta el viento pueden hacer desaparecer toda evidencia de la falla [9], [10], [11].

\subsection{Limpieza de superficie de fractura}

La Fig. 9 muestra el efecto de la limpieza ultrasónica en la restauración de las superficies de fracturas, cabe resaltar que factores tales como concentración de la solución y tipo (ácida o alcalina), números de ciclos de lavado influyen en el resultado final.

Fig. 9. EFECTO DE LA LIMPIEZA ULTRASÓNICA EN LA RECUPERACIÓN DE SUPERFICIES DE FRACTURA ANTES (A) DESPUÉS (B). LIMPIEZA CON UN CICLO 460 SEG
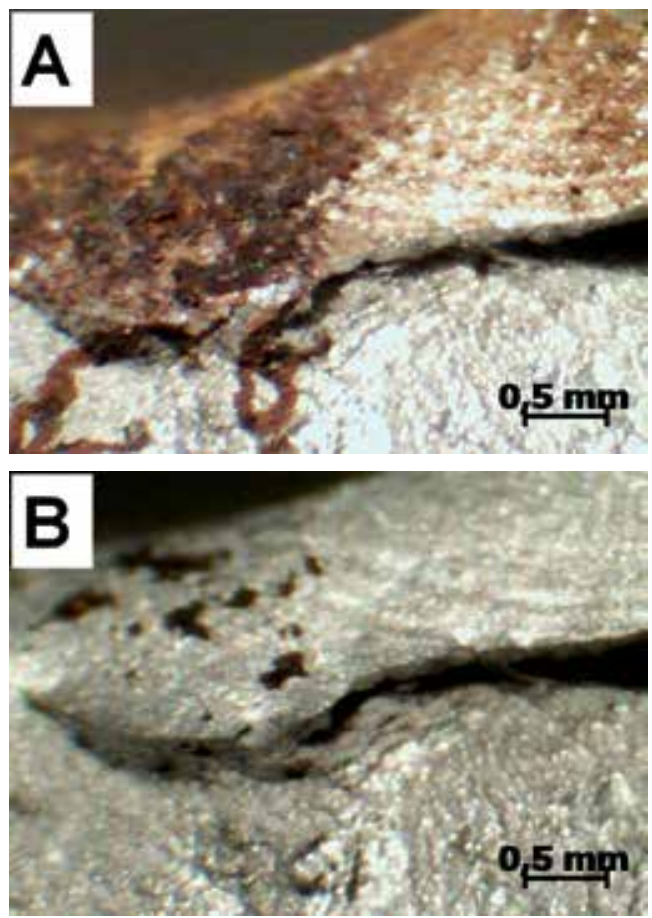

Fuente: autor.

Este método no se recomienda para superficies de fallas que requieran preservar los productos de corrosión para su evaluación, por lo que la limpieza debe ser ejecutada siempre y cuando se desee conocer los rasgos de la fractura y el avance de la grieta.

Por otro lado, si se desea conocer la morfología de los productos de corrosión que fueron afectadas por el medio ambiente, estas no darán resultados positivos, ya que los depósitos aportados por el medio ambiente se pueden confundir con los generados por el medio corrosivo donde esta- 
ba ubicada la pieza, de ahí que, la preservación de las muestras sigue siendo vital para el éxito de una investigación de fallas [12].

\section{CONCLUSIONES}

El medio ambiente es el más perjudicial de una fractura, ya que este aporta los elementos corrosivos que permiten acelerar el crecimiento de los productos de corrosión.

La corrosión depende de la condición superficial de la fractura mientras menos rugosa sea la fractura más lenta será la oxidación

La limpieza ultrasónica puede contribuir a restaurar parte de la superficie original fracturada de la pieza, sin embargo, no es recomendable para cuando el interés del analista es evaluar productos de corrosión.

\section{REFERENCIAS}

[1] M. Pepi, "Sample preservation - the key to a successful failure analysis," Sept. 2008. Recuperado de http:// www.dtic.mil/cgi-bin/GetTRDoc?AD=ADA493766.

[2] D. Button, "Evidence Collection, Preservation, and Chain of Custody," National Association of Subrogation Professionals "Subrogation" Magazine, 2009. Recuperado de https://www.google.com.co/url?sa=t\&rct$=j \& q=\& e s r c=s \&$ source $=$ web $\& c d=1 \& c a d=r j a \&$ uact $=$ 8\&ved=0ahUKEwjZvYiVit_NAhVCKh4KHdgQD2UQFg gkMAA\&url=http\%3A\%2F\%2Fwww.nelsonforensics. com\%2FDownloads\%2FEvidence_Storage.pdf\&usg=A FQjCNEuiXKAugIYdwJIJekTFCe8kgByiQ

[3] M.Mullen, A. Griebel y J. Tartaglia, "Fracture Surface analysis", Revista Advanced Materials \& Processes. Dec. 2007. Recuperado de https://www.google.com.co/url?sa=t\&rct$=j \& q=\& e s r c=s \&$ source $=$ web $\& c d=1 \& c a d=r j a \&$ uact $=$ $8 \&$ ved=0ahUKEwjFsNOIx-HNAhULqh4KHZQ1AxkQFg gqMAA\&url=http\%3A\%2F\%2Fwww.asminternational. org\%2Fdocuments\%2F10192\%2F1894865\%2Fam p16512p021.pdf\%2F2732e95c-08c6-4b71-a321$488356641445 \% 2$ FAMP16512P021\&usg $=A F Q j C N E j R P s c 2$ wsD3xhjy9Z0mASzrKBnQ\&bvm=bv.126130881,d.dmo
[4] Y. Guevara, "Análisis de falla del eje agotador del reactor 406 de la empresa Tripoliven," Pregrado, Dept. Met. Instituto Universitario de Tecnología Puerto CabeIlo, Venezuela, 2013.

[5] S. Mukhopadhyay, "Sample Preparation Techniques for Characterization of Solid Surfaces and Films," Sample Preparation Techniques in Chemical Measurements, Edited by S. Mitra, John Wiley \& Sons, 2003. Recuperado de https://www.google.com.co/url?sa=t$\& r c t=j \& q=\& e s r c=s \&$ source $=$ web $\& c d=2 \& c a d=r j a \& u a$ $\mathrm{ct}=8 \& \mathrm{ved}=0$ ahUKEwiOqMPDhN_NAhWE8x4KHda4C fYQFggmMAE\&url=http\%3A\%2F\%2Fwww.spectroscopynow.com $\% 2$ Fuserfiles $\% 2$ Fsepspec\%2Ffile $\% 2$ Fspec NOW\%2FTutorials\%2Fsample_prep_mitra_377-412. pdf\&usg=AFQjCNH3sKiSZ9r9gjjojcW9dZsyNoFkow

[6] ASM Metals Handbook: “Fractography”, vol. 12, ASM International, Materials Park ASM International. Park, Ohio, p. 141, 2004.

[7] ASTM E-23, "Standard Method for Notched Bar Impact Testing of Metallic Materials," 1982.

[8] Gil, L., “Corrosión Atmosférica," L. Gil (comps.). Fundamentos de Corrosión, pp. 159-170, 2000. Venezuela: Universidad Experimental Politécnica Antonio José de Sucre.

[9] ASM Metals Handbook: "Corrosion", vol. 13, ASM International, Materials Park ASM International. Park, Ohio, pp. 2406-2410, 2004.

[10] C Pereira, "Evaluación del comportamiento del acero estructural ASTM A-36 con recubrimiento de pintura anticorrosivo epoxica y alquídica en el ambiente marino de la empresa pequiven y con el equipo de niebla salina del IUTPC," Pregrado, Dept. Met. Instituto Universitario de Tecnología Puerto Cabello, Venezuela, 2013.

[11] L. Gil, V. Maizo, O. León, M. Martínez, L. D`ónofrio y F. Gómez, "Evaluación de la resistencia a la corrosión atmosférica de los aceros Siderúrgica del Orinoco (SIDOR) ARCO (A-588) y acero al carbono (A-36) en medio rural," Revista Universidad, Ciencia y Tecnología, Año 1, no. 2, pp. 30-36, Jun. 1997.

[12] ASM Metals Handbook: "Failure Analysis and Prevention”, vol. 11, ASM International, Materials Park ASM International. Park, Ohio, p. 835, 2005. 